\title{
Single nucleotide polymorphism of NLRP3 (Q705K) In juvenile spondyloarthritis and oligo/ polyarticular juvenile idiopathic arthritis
}

\author{
Marija Perica ${ }^{1 *}$, Mandica Vidovic ${ }^{1}$, Lovro Lamot ${ }^{1,2}$, Lana Tambic Bukovac ${ }^{1}$, Sanja Kapitanović ${ }^{3}$, Miroslav Harjaček ${ }^{1}$ \\ From 21st European Pediatric Rheumatology (PReS) Congress \\ Belgrade, Serbia. 17-21 September 2014
}

\begin{abstract}
Introduction
The NLRP3 inflammasome is a key component of the innate immune system serving as an intracellular sensor of microbial components and cell injury. Gain-of-function mutations of the NLRP3 gene, such as single nucleotide polymorphism (SNP) Q705K, lead to autoproteolytic activation of caspase 1 , resulting in excessive and uncontrolled production of proinflammatory cytokines. This may represent the mechanism by which an inflammatory loop is triggered leading to a long-term inflammatory phenotype.
\end{abstract}

\section{Objectives}

Our objective was to compare the frequency of SNP Q705K of NLRP3 among patients with juvenile spondyloarthritis (jSpA) and juvenile idiopathic arthritis (JIA).

\section{Methods}

DNA was extracted from blood samples of $37 \mathrm{jSpA}$ patients and patients with oligoarticular or polyarticular JIA, diagnosed according to ILAR criteria. Polymorphism of the NLRP3 (Q705K) was determined using real time and multiplex PCR.

\section{Results}

Among 37 genotyped patients, 24 patients with jSpA (92.31\%) and 9 patients (81.82\%) with JIA were carriers of the wild type allele. Only 2 patients in each group were heterozygous for NLRP3 (Q705K) polymorphism (7.69\% in jSpA and $18.18 \%$ in JIA group). Although the observed frequency among groups was not statistically significant (Pearson Chi-square 0.8820724, $\mathrm{p}=0.64337$ ), the frequency of allele polymorphism observed among our study population was higher (10.81\%) than previously described in the general population (6.5\%).

\section{Conclusion}

The frequency of SNP Q705K of the NLRP3 gene did not differ among jSpA and JIA patients. There was also no evidence that variant of NLRP3 is a major risk factor for jSpA or JIA, however, lack of susceptibility should be confirmed in a larger group of patients.

\section{Disclosure of interest}

None declared.

\section{Authors' details}

${ }^{1}$ Pediatric and Adolescent Rheumatology, Children's Hospital Srebrnjak, Croatia. ${ }^{2}$ University of Zagreb School of Medicine, Zagreb, Croatia. ${ }^{3}$ The Ruđer Bošković Institute, Zagreb, Croatia.

Published: 17 September 2014

doi:10.1186/1546-0096-12-S1-P31

Cite this article as: Perica et al:: Single nucleotide polymorphism of NLRP3 (Q705K) In juvenile spondyloarthritis and oligo/ polyarticular juvenile idiopathic arthritis. Pediatric Rheumatology 2014 12(Suppl 1):P31.

'Pediatric and Adolescent Rheumatology, Children's Hospital Srebrnjak,

Croatia

Full list of author information is available at the end of the article 